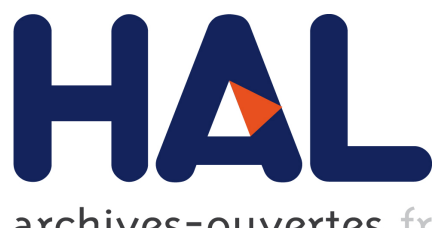

archives-ouvertes

\title{
The finiteness of logic programming derivations Philippe Balbiani
}

\section{To cite this version:}

Philippe Balbiani. The finiteness of logic programming derivations. Third International Conference on Algebraic and Logic Programming, Sep 1992, Volterra, Italy. hal-03252315

\section{HAL Id: hal-03252315 \\ https://hal.archives-ouvertes.fr/hal-03252315}

Submitted on 7 Jun 2021

HAL is a multi-disciplinary open access archive for the deposit and dissemination of scientific research documents, whether they are published or not. The documents may come from teaching and research institutions in France or abroad, or from public or private research centers.
L'archive ouverte pluridisciplinaire HAL, est destinée au dépôt et à la diffusion de documents scientifiques de niveau recherche, publiés ou non, émanant des établissements d'enseignement et de recherche français ou étrangers, des laboratoires publics ou privés. 


\title{
The finiteness of logic programming derivations
}

\author{
Philippe Balbiani $(l)$
}

Institut de Recherche en Informatique de Toulouse

\begin{abstract}
The question of the termination of logic programming computations is studied from a semantical point of view. To every program are associated two first order formulas. Their valid consequences are respectively the finiteness and the infiniteness SLDNF sets of the logic programs considered. The non-existence of a recursive safe computation rule leading into an infinite $S L D N F$ computation is proved.
\end{abstract}

\section{Introduction}

When does it stop? This question is common to many fields in computer science. To ask it in a logic programming setting does not make it easier. the restriction of first order predicate calculus to definite Horn clauses has the full power of recursion theory. Thus, because of the indecidability of the halting problem, there is no procedure to decide whether a computation will end or not. Non-termination is one of the most inefficient behaviour of programs. To force termination, programmers sometimes decide to insert control informations in their programs. This leads to imperative programs: from the point of view of logic programming, this is not an ideal option. Another way to limit the number of non-terminating execution is to allow the interpreter to cut some derivations [3] or to forbide multiple use of some clauses. Other methods consist in the analysis of the stream of informations between clauses and in the resolution of a linear system of equations [16]. For some classes of programs, these methods provide decision procedures for termination $[1,3,7,16,18]$.

We do not propose here another method to decide termination in logic programming. We rather propose a semantical characterization of finite and infinite SLDNF computations.

To every program is firstly associated a first order classical formula: the completion of finiteness. As Clark's formula [10] offered a characterization of SLDNF finite failure, the ground valid consequences of this completion formula of finiteness will be the finiteness set of the logic program considered, that is to say: the set of ground atoms from which there cannot be any infinite SLDNF computation.

Secondly, a completion formula of infiniteness is defined. This formula will belong to 
the language of first order modal logic of provability. Its ground valid consequences are the set of ground atoms from which it is possible to have an infinite SLDNF computation. Since the modal logic of provability [9] is not axiomatizable, this characterization does not lead to a decision procedure for infinite SLDNF computation. Consequently, we ask the question of the existence of an algorithm leading, every time it is possible, to an infinite computation. It is proved that such an algorithm cannot exist: for some logic programs, a safe computation rule leading to an infinite derivation every time it is possible cannot be recursive.

\section{Normal Logic Programs}

The first order language in which normal logic programms will be written, $\mathscr{L}$, is made of variables, constants, function symbols, predicate symbols ${ }^{(2)}$, classical connectors $\neg$, $\wedge, \vee$ and $\leftarrow$, the quantifiers $\exists$ and $\forall$ and the usual punctuation symbols. Preinterpretation, interpretation, variable assignment (with respect to some preinterpretation), term assignment (with respect to some pre-interpretation and variable assignment) are defined as usual (see Lloyd [14] for precise definitions). Let $J$ be a preinterpretation of $\mathscr{L}, V$ a variable assignment with respect to $J$ and $A$ an atom. We suppose that $A$ is of the form $p\left(t_{1}, \ldots, t_{n}\right)$ and that $d_{1}, \ldots, d_{n}$, elements of the domain of $J$, are the term assignments of $t_{1}, \ldots, t_{n}$. We call $A_{J, V}=p\left(d_{1}, \ldots, d_{n}\right)$ the $J$-instance of $A$ with respect to $V$. Let $[A]_{J}=\left\{A_{J, V}: V\right.$ is a variable assignment with respect to $\left.J\right\}$. Let $I$ be an interpretation of domain $D$ of $\mathscr{L}$ and $V$ a variable assignment. To every formula of $\mathscr{L}$ is given a truth value, true or false, (with respect to $I$ and $V$ ) following the classical rules of first order predicate calculus. A ground term (atom) is a term (atom) without variable. The Herbrand universe $U_{\mathscr{L}}$ is the set of ground terms of $\mathscr{L}$. The Herbrand base $B_{\mathscr{L}}$ is the set of ground atoms of $\mathscr{L}$. The Herbrand pre-interpretation is the pre-interpretation such that: (a) its domain is $U_{\mathscr{L}}$, (b) constants are assigned to themselves, (c) the mapping from $\left(U_{\mathscr{L}}\right)^{n}$ to $U_{\mathscr{L}}$ defined with $\left(t_{1}, \ldots t_{n}\right) \rightarrow f\left(t_{1}, \ldots t_{n}\right)$ is assigned to any function symbol $f$ of arity $n$. An Herbrand interpretation is an interpretation based on the Herbrand pre-interpretation.

Normal clause, normal program, normal goal, derived goal, safe computation rule, $S L D N F$ derivation, $S L D N F$ refutation and $S L D N F$ tree are defined as in [14]. A safe computation rule always selects, if possible, a positive or a ground negative literal in a 
given goal. An SLDNF computation flounders if this selection is not possible. Let $P$ be a normal program and $G$ be a normal goal. The finiteness set of $P$ is the set of all $A \in B_{\mathscr{L}}$ such that there is no infinite $S L D N F$ computation of $P \cup\{\leftarrow A\}$. The substitution $\theta$ is a computed answer of finiteness of rank $n$ of $P \cup\{G\}$ if there is no $S L D N F$ computation of $P \cup\{G \theta\}$ involving $n$ or more than $n$ goals. The substitution $\theta$ is a computed answer of finiteness of $P \cup\{G\}$ if there is no infinite $S L D N F$ computation of $P \cup\{G \theta\}$. The infiniteness set of $P$ is the set of all $A \in B_{\mathscr{L}}$ such that there is an infinite SLDNF computation of $P \cup\{\leftarrow A\}$. The substitution $\theta$ is a computed answer of infiniteness of rank $n$ of $P \cup\{G\}$ if there is an $S L D N F$ computation of $P \cup\{G \theta\}$ involving $n$ or more than $n$ goals. The substitution $\boldsymbol{\theta}$ is a computed answer of infiniteness of $P \cup\{G\}$ if there is an infinite $S L D N F$ computation of $P \cup\{G \theta\}$.

\section{A Completion Formula}

We define in this section a completion formula of finiteness for all normal programs. As Clark's formula [10], it will be obtained putting on the same side of an implication symbol the bodies of the clauses defining some predicate. Let $p\left(t_{1}, \ldots, t_{n} k-L_{1}, \ldots, L_{m}\right.$ be a clause of a program $P$. We will need a new predicate symbol: the equality predicate, $=$. The first step is to transform this clause into the formula $p\left(x_{1}, \ldots x_{n}\right) \leftarrow\left(L_{1} \wedge \ldots \wedge L_{m} \leftarrow\left(x_{1}=t_{1}\right) \wedge \ldots \wedge\left(x_{n}=t_{n}\right)\right)$ where the variables $x_{1}, \ldots, x_{n}$ do not appear in $P$. If $y_{1}, \ldots, y_{d}$ are the variables of the original clause then this formula is transformed into $p(x l, \ldots . . x n) \leftarrow \forall y 1 \ldots \forall y d\left(L_{1}{ }^{*} \wedge . . . \wedge L_{m}{ }^{*} \leftarrow\left(x_{1}=t_{1}\right) \wedge \ldots \wedge\left(x_{n}=t_{n}\right)\right)$, where: if $L_{i}$ is an atom then $L_{i}=L_{i}^{*}$ else $L_{i}$ is a negative literal and $L_{i}=\neg L_{i}^{*}$. Let us suppose this transformation has been done for every clause appearing in the definition of $p$. Then we have $k$ formulas of the form $p\left(x_{1}, \ldots, x_{n}\right) \leftarrow-E_{1}, \ldots, p\left(x_{1}, \ldots, x_{n}\right) k E_{k}$, where each $E_{i}$ is of the form $\forall y_{1} \ldots \forall y_{d}\left(L_{1} * \wedge . . \wedge L_{m}{ }^{*} \leftarrow\left(x_{1}=t_{1}\right) \wedge . . \wedge\left(x_{n}=t_{n}\right)\right)$. The completed definition of finiteness of $p$ is then the formula $\forall x_{1} \ldots \forall x_{n}\left(p\left(x_{1}, \ldots, x_{n}\right) \leftarrow E_{1} \wedge \ldots \wedge E_{k}\right)$.

It might be the case that some predicate symbols are not the head of any clauses in $P$. For such a predicate symbol $q$, we explicitly add the formula $\forall x_{1} \ldots \forall x_{n} q\left(x_{1}, \ldots, x_{n}\right)$. This formula is also called the completed definition of finiteness of $q$. Let $P$ be a nomal program. The completion of finiteness of $P, \operatorname{comp}_{F}(P)$, is the collection of all the completed definition of finiteness of the predicate symbols of $\mathscr{L}$ together with CET, 
Clark's equational theory that defines the predicate symbol of equality [10]. Our completion of finiteness possesses some properties possessed by $P$ itself. Next result, for example, states that the set of Herbrand models of $\operatorname{comp}_{F}(P)$ is a complete lattice.

proposition 3.1 Let $P$ be a normal logic program and $\left\{M_{i}\right\}_{i \in I}$ a non-empty set of Herbrand models of $\operatorname{comp}_{F}(P)$. Then $\cap_{i \in I^{M}}$ is a Herbrand model of comp $F_{F}(P)$.

Thus, since $B_{\mathscr{L}}$ is a Herbrand model of $\operatorname{comp}_{F}(P)$, the intersection $M_{P}$ of all Herbrand models of $\operatorname{comp}_{F}(P)$ is still a model of $\operatorname{comp}_{F}(P)$. It is the least Herbrand model of $\operatorname{comp}_{F}(P)$. Unfortunately, it is not true that: $M_{P}=\left\{A \in B_{\mathscr{L}}: A\right.$ is a valid consequence of $\left.\operatorname{comp}_{F}(P)\right\}$. As a matter of fact, if $P=\{A \leftarrow B(x), B(f(x)) \leftarrow B(x)\}$ then the least Herbrand model of $\operatorname{comp}_{F}(P)$ is $M_{P}=\{A\} \cup\{B(a), B(f(a)), \ldots\}$ but $A$ is not a valid consequence of $C E T \cup\{A \leftarrow \forall x B(x), \forall y(B(y) \leftarrow \forall x(B(x) \leftarrow y=f(x)))\}$.

To the procedural notion of a computed answer of finiteness is associated the semantical notion of a correct answer. Let $P$ be a normal program, $G$ the normal goal $\leftarrow L_{1}, \ldots, L_{n}$ and $\theta$ a substitution of the variables of $G$. We say that $\theta$ is a correct answer for $\operatorname{comp}_{F}(P) \cup\{G\}$ if $\forall\left(\left(L_{1}{ }^{*} \wedge . . . \sim L_{n}{ }^{*}\right) \theta\right)$ is a valid consequence of $\operatorname{comp} p_{F}(P)$. Theorem 3.5 will give a first relation between computed answers of finiteness and correct answers.

Now we define a mapping $T_{P}^{J}$ on the lattice of interpretarions (based on some preinterpretation $J$ of the language) to itself. If $J$ is a pre-interpretation of $\mathscr{L}$ and $I$ is an interpretation based on $J$ then $T_{P}^{J}(I)=\{B$ : for every variable assignment $V$ with respect to $J$ and for every clause $A \leftarrow L_{1}, \ldots, L_{n}$ in $P_{n}$ if $A_{J, V}=B$ then $L_{1}^{*} \wedge \ldots L_{n}{ }^{*}$ is true with respect to $I$ and $V$ \}. When $J$ is the Herbrand pre-interpretation of $\mathscr{L}$, we will write $T_{P}$ instead of $T_{P}{ }^{J}$. The mapping $T_{P}{ }^{J}$ possesses the usual properties.

proposition 3.2 For every pre-interpretation $J$ of $\mathscr{L}$ and for every normal program $P$, the mapping $T_{P}^{J}$ is monotonic.

The mapping $T_{P}{ }^{J}$ is not always continuous. Nevertheless its pre-fixpoints are models of the completion of finiteness of the program considered. 
proposition 3.3 Let $P$ be a normal program, $J$ a pre-interpretation of $\mathscr{L}$ and $I$ an interpretation based on $J$. Let us suppose that $I$, together with the identity relation assigned to $=$, is a model of the equality theory. We have: I, together with the identity relation assigned to $=$, is a model of $\operatorname{comp}_{F}(P)$ iff $T_{P}^{J}(I)$ ㄷ․

As a corollary, we have:

proposition 3.4 The least Herbrand model of comp f $_{F}(P)$ is the least fixpoint of $T_{P}$.

Note that, for every program $P, g f p\left(T_{P}\right)=B \mathscr{L}$. A first important result is the soundness of our completion formula for $S L D N F$ resolution (see theorem 3.5 below). Let $P$ be a normal program and $G$ be a normal goal. We say that $P \cup\{G\}$ is allowed whenever no $S L D N F$ computation of $P \cup\{G\}$ flounders. We say that $P$ is allowed whenever, for every ground atom $A$, no $S L D N F$ computation of $P \cup\{\leftarrow A\}$ flounders.

theorem 3.5 Let $P$ be a normal program and $G$ be a normal goal. If $P \cup\{G\}$ is allowed then every computed answer of finiteness of $P \cup\{G\}$ is a correct answer of $\operatorname{comp}_{F}\{P) \cup\{G\}$.

proof Let $\theta$ be a computed answer of finiteness of $P \cup\{G\}$. Since there is no infinite $S L D N F$ computation of $P \cup\{G \theta\}$, there is an integer max greater than the number of goals involved in any $S L D N F$ computation of $P \cup\{G \theta\}^{(3)}$. The induction on $\max$ is straightforward.

As a corollary, we have:

corollary 3.6 Let $P$ be a normal program and $G$ a normal goal. If $P \cup\{G\}$ is allowed and if there is no infinite SLDNF computation of $P \cup\{G\}$ then the empty substitution is a correct answer for comp $F(P) \cup\{G\}$.

Let $P$ be a normal program. A consequence of corollary 3.6 is the inclusion of the set of finiteness of $P$ in the least Herbrand model of $\operatorname{comp}_{F}(P)$. However, it is not always equal to this least Herbrand model. Let us consider the program $P=\{A \leftarrow B(x)$, $B(f(x)) \leftarrow B(x)\}$. The least Herbrand model of $\operatorname{comp}_{F}(P)$ is $M_{P}=\{A\} \cup\{B(a), B(f(a)), \ldots\}$ but there is an infinite $S L D N F$ computation of $P \cup\{\leftarrow A\}$. For that program, $T_{P} \uparrow \omega=$ 
$\{B(a), B(f(a)), \ldots\}$ and $M_{P}=T_{P} \uparrow \omega+1$. In other respects, if $A \in B \mathscr{L}$, if $P \cup\{\leftarrow A\}$ is allowed and if there is no $S L D N F$ computation of $P \cup\{\leftarrow A\}$ involving $n$ or more than $n$ goals then $A \in T_{P} \uparrow \omega$. If $A$ is an atom, we define $[A]=\left\{A^{\prime} \in B \mathscr{L}: A^{\prime}=A \theta\right.$, for some substitution $\theta]$. Then, $[A]$ is the set of ground instances of $A$.

theorem 3.7 Let $P$ be a normal program and $G$ the normal goal $\leftarrow L_{1}, \ldots, L_{m}$. If $P \cup\{G\}$ is allowed and if $\theta$ is a computed answer of finiteness of rank $n$ of $P \cup\{G\}$ then $\cup_{j=1 . . m}\left[L_{j}^{*} \theta\right] \subseteq T_{P} \uparrow \omega$.

proof The proof is a straightforward induction on the rank of the computed answer.

As a corollary, we have:

corollary 3.8 The set of finiteness of $P$ is included in $T_{P} \uparrow \omega$.

The set of computed answer of finiteness of $P \cup\{G\}$ is not always finite (just consider the program containing one clause: $A(f(x)) \leftarrow A(x))$. The possibility of its finiteness will not be studied here. Now we give the first completeness result of the finiteness of SLDNF resolution. Its proof is similar to the completeness proof of the negation as failure rule given by Lassez, Maher and Wolfram [13].

theorem 3.9 Let $P$ be a normal program and $G$ a normal goal. Every correct answer for comp $F_{F}(P) \cup\{G\}$ is a computed answer of finiteness of $P \cup\{G\}$.

proof Suppose there is an infinite $S L D N F$ computation of $P \cup\{G\}$. We show the empty substitution is not a correct answer for $\operatorname{comp}_{F}(P) \cup\{G\}$. Let $G_{0}=G=\leftarrow l_{1}, \ldots, l_{m}, G_{1}, \ldots$ be the infinite $S L D N F$ computation of $P \cup\{G\}$. Let $\theta_{1}, \theta_{2}, \ldots$ be the $m g u$ and $C_{1}, C_{2}$, ... the input clauses of this derivation. Let ${ }^{\circ}$ be the relation defined on terms by $s^{\circ} t$ if and only if there is an integer $n$ such that $s \theta_{2} \ldots \theta_{n}=t \theta_{1} \ldots \theta_{n}$. Of course, ${ }^{\circ}$ is an equivalence relation on the set of terms of the language. For every term $t$, we note [ $t$ ] its class modulo ${ }^{\circ}$. Let $D$ be the set of equivalence classes modulo ${ }^{\circ}$. Let $J$ be the preinterpretation of $\mathscr{L}$ with domain $D$ assigning to each constant $c$ its class $[c]$ and assigning to each function symbol $f$ of arity $n$ the function from $D^{n}$ to $D$ defined by: $\left(\left[s_{1}\right], \ldots,\left[s_{n}\right]\right) \rightarrow\left[f\left(s_{1}, \ldots, s_{n}\right)\right]$. Let $I$ be the interpretation based on $J$ defined by: $I=$ 
$\left\{p\left(\left[t_{1}\right], \ldots,\left[t_{n}\right]\right):\right.$ for every element $t_{1}^{\prime}, \ldots, t_{n}$ in $\left[t_{1}\right], \ldots,\left[t_{n}\right]$, the set of proper successors of $p\left(t^{\prime}{ }_{1}, \ldots, t_{n}\right)$ in the computation is finite). We show that $T_{P}^{J}(I) \subseteq I$. If $p\left(\left[t_{1}\right], \ldots,\left[t_{n}\right]\right) \in I$ then there are elements $t^{\prime} 1, \ldots, t_{n}^{\prime}$ in $\left[t_{1}\right], \ldots,\left[t_{n}\right]$ such that the set of the proper successors of $p\left(t^{\prime} 1, \ldots, t_{n}\right)$ in the computation is infinite. Consequently, there is an integer $i_{0}$ such that the goal $G_{i 0}$ contains $p\left(t^{\prime}{ }_{1}, \ldots, t_{n}\right)$ as a subgoal, there is an integer $i$ greater or equal to $i_{0}$, there is a clause $C_{i+1}=p\left(s_{1} \ldots, s_{n}\right) k-L_{1}, \ldots, L_{m}$ in $P$, a substitution $\theta_{i+1}$ and an integer $j$ in $1, \ldots, m$ such that $\theta_{i+1}=$ $m g u\left(p\left(t^{\prime}{ }_{1} \theta_{i 0+1} \ldots \theta_{i}, \ldots, t_{n}^{\prime} \theta_{i 0+1} \ldots \theta_{i}\right), p\left(s_{1}, \ldots, s_{n}\right)\right)$ and the set of proper successors of $L_{j} \theta_{i+1}$ in the computation is infinite. Consequently, $\left[t_{1}\right]=\left[s_{1}\right], \ldots,\left[t_{n}\right]=\left[s_{n}\right]$ and there is a clause $A \leftarrow L_{1}, \ldots, L_{m}$ in $P$ and a variable assignment $V$ with respect to $J$ such that $A_{J, V}=p\left(\left[t_{1}\right], \ldots,\left[t_{n}\right]\right)$ and $L^{*}{ }_{1} \wedge \ldots \wedge L^{*}{ }_{m}$ is false with respect to $I$ and $V$.

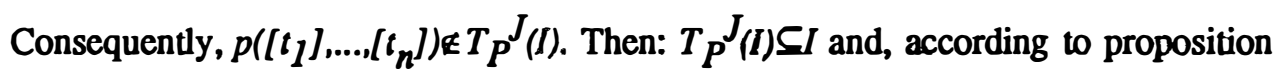
3.3, I is a model of $\operatorname{comp}_{F}(P)$. In others respects, it is not difficult to show that $I$ is not a model of $\forall\left(l^{*}{ }_{1^{\wedge} . . . \wedge l^{*}}{ }_{m}\right)$. Thus the empty substitution is not a correct answer for $\operatorname{comp}_{F}(P) \cup\{G\}$.

The following theorem states the point of view of the fixpoint operator about the completeness of the finiteness of SLDNF derivation.

theorem 3.10 Let $P$ be a normal program and $G$ the nomal goal $\leftarrow L_{1}, \ldots, L_{m}$. If $\cup_{j=1 . . m}\left[L_{j}{ }^{*} \theta\right] S_{P} T_{P}$ in then $\theta$ is a computed answer of finiteness of rank $n$ of $P \cup\{G\}$.

proof By induction on the integer $n$.

As a corollary we have:

corollary 3.11 If $P$ is allowed then the set of finiteness of $P$ is equal to $T_{P} \uparrow \omega$.

\section{Infinite Derivations}

Connectors used so far were truth-functional: the truth value of every formula formed by them only depends on the truth values of its subformulas. Now, our language will include a pair of intensional connectors (the modal connectors 
$\square$ and $\diamond$ ) which will be no more truth-functional. Our language contains the following rule: if $F$ is a formula then so is $\square F$. The connector $\diamond$ is defined by: $\diamond F=\operatorname{def} \square \neg F$. We could give to $\square$ and $\diamond$ a variety of interpretations. Historically, modal logic is the logic of possibility and necessity: $\square F$ and $\diamond F$ are usualy read " $F$ is necessary" and " $F$ is possible". For us, $\square$ will be used to denote the temporal relationship between bodies and heads of definite clauses. For example, we will formally represent through the modal fornula $\square(A \leftarrow \square B)$ the procedural role of the clause $A \leftarrow B$.

A pre-interpretation of our first order modal language is made of: a non-empty set $D$, the domain; to each constant in $\mathscr{L}$ the assignment of an element in $D$; to each function symbol of arity $n$, the assignment of a function from $D^{n}$ to $D$; a non-empty set $M$, the universe or set of possible worlds of the pre-interpretation; a binary relation $R$ on $M$, the accessibility relation between possible worlds. We will require that this accessibility relation is transitive and reverse well-founded.

note This condition of well-foundedness is of fundamental importance for us. As a matter of fact, we will have to prove the equivalence between " $\square A$ is a valid consequence of $\operatorname{comp}_{I}(P)$ " and "there is an $S L D N F$ computation of $P \cup\{\leftarrow A\}$ involving an infinite number of goals", comp $(P)$ being some modal completion of infiniteness of $P$. On one hand, we will prove by induction on the longest $S L D N F$ derivation of $P \cup\{\leftarrow A\}$ that $\square A$ is not a valid consequence of $c \operatorname{comp}_{I}(P)$. On the other hand, we will prove by induction on the model of $\operatorname{comp}_{\lambda}(P) \cup\{\neg \square A\}$ that there is no infinite $S L D N F$ computation of $P \cup\{\leftarrow A\}$. This last induction holds because the accessibility relation between the possible worlds of a model of $\operatorname{comp}_{\mathbb{R}}(P) \cup\{\neg \square A\}$ is well-founded.

An interpretation I of a first order modal language $\mathscr{L}$ over a pre-interpretaion $J$ with domain $D$ and universe $M$ is made of: for every predicate symbol of arity $n$, the assignment of a function from $M x D^{n}$ to $\{$ true, false $\}$. We thus say that $I$ is based on $J$. Let $J$ be a pre-interpretation with domain $D$ and universe $M$. Let $I$ be an interpretation based on $J$ and $V$ a variable assignment. To every possible world and formula can be atributed a truth value, true or false, (with respect to $I$ and $V$ ) as follows: if the formula is of the forn $\square F(\diamond F)$ then its truth value in $w$ is true if and only if, in every (some) possible world accessible from $w$ using $R$, the truth value of $F$ is true. The Herbrand pre-interpretation is a pre-interpretation whose domain is $U_{\mathscr{L}}$. A Herbrand 
interpretation of $\mathscr{L}$ is an interpretation based on the Herbrand pre-interpretation.

A formula is sarifiable (valid) if it is true in some (every) possible world of some (every) interpretation. It is a valid consequence of some set of formulas if it is tre in every possible world (of every interpretation) satisfying every formula of this set. Let us consider the set of valid formulas. It is not recursively enurnerable. As a matter of fact, validity (in the transitive and reverse well-founded interpretations we are considering) is highly indecidable: it is $\Pi_{2}{ }^{1}$-complete in the analytical hierarchy [9]. If the language is restricted to its propositional part then one gets $\mathrm{Pr}$, the (decidable) propositional modal logic of provability. Its axiom schemata and inference rules are those of the classical propositional calculus plus:

(a) $(\square A \leftarrow \square B) \leftarrow \square(A \leftarrow B)$.

(b) $\square A \leftarrow \square(A \leftarrow \square A)$.

(c) if $\vdash{ }_{P r} A$ then $\vdash{ }_{P r} \square A$.

This modal logic is of importance because of its relationship with provability in arithmetic. For further informations, we suggest the reader consult the book by Boolos [8]. As far as we know, the following results together with the previous ones presented in [4] and [5] are the first use of this modal logic for the semantical characterization of a programming language.

In [4] was defined a modal completion formula $\operatorname{comp}_{C W A}(P)$ of any definite logic program $P$. It was proved that there was no $S L D$ refutation of $P \cup\{\leftarrow A\}$ if and only if $\square A$ is a valid consequence of $\operatorname{comp}_{C W A}(P)$ in transitive and reverse well-founded interpretations. In [5] was defined a modal completion formula $\operatorname{comp}_{D^{(P)}}$ of any normal logic program $P$. It was proved that if the program is stratified then $A$ belongs to its natural interpretation as it has been defined in [2] if and only if $\square A$ is a valid consequence of $\operatorname{comp}_{D}(P)$ in transitive and reverse well-founded interpretations.

We would like to define a modal completion formula comp $p_{(}(P)$ of any normal logic program $P$ such that there is an infinite $S L D N F$ computation of $P \cup\{\leftarrow A\}$ if and only if $\square A$ is a valid consequence of $c o m p_{I}(P)$ in transitive and reverse well-founded interpretations.

Let $p\left(t_{1}, \ldots, t_{n}\right) \leftarrow L_{1}, \ldots, L_{m}$ be a clause of a normal program $P$. The first step is to transform it into the formula $p\left(x_{1}, \ldots . x_{n}\right) \leftarrow\left(\left(\square L_{1} \vee \ldots \vee v L_{m}\right) \wedge\left(x_{1}=t_{1}\right) \wedge \ldots \wedge\left(x_{n}=t_{n}\right)\right)$ where the variables $x_{1}, \ldots, x_{n}$ do not appear in $P$. If $y_{1}, \ldots, y_{d}$ are the variables of the original clause then we transform this formula into the formula $p\left(x_{1}, \ldots, x_{n}\right) \leftarrow \exists y_{1} \ldots \exists y_{d}$ 
$\left(\left(\square L_{1}^{*} \vee \ldots \vee \square L_{m}{ }^{*}\right) \wedge\left(x_{1}=t_{1}\right) \wedge . . \wedge\left(x_{n}=t_{n}\right)\right)$. Now suppose this transfornation has been made for every clause in the definition of $p$. Then we have $k$ transformed clauses of the form $p\left(x_{1}, \ldots, \hbar\right) \leftarrow E_{1}, \ldots, p\left(x_{1}, \ldots x_{n}\right) \leftarrow E_{k}$ where each $E_{i}$ is of the forn $\exists y_{1} . \exists y_{d}$ $\left(\left(\square L_{1}^{*} \vee \ldots \vee \square L_{m}^{*}\right) \wedge\left(x_{1}=t_{1}\right) \wedge . . . \wedge\left(x_{n}=t_{n}\right)\right)$. Then, the completed definition of infinitene ssof $p$ is the formula $\forall x_{1} \ldots \forall x_{n} \square\left(p\left(x_{1}, \ldots, x_{n}\right) \leftarrow E_{1} \vee \ldots v E_{k}\right)$.

Furthemore we add the following modal equational theory:

1. $\square(c \neq d)$,for every pair $c, d$ of distinct constants.

2. $\square\left(f\left(x_{1} \ldots, x_{n}\right) \neq g\left(y_{1}, \ldots, y_{n}\right)\right)$,for every pair $f, g$ of distinct function symbols.

3. $\square\left(f\left(x_{1}, \ldots, x_{n}\right) \neq c\right)$, for every constant $c$ and every function symbol $f$.

4. $\mathrm{Q}(t[x] \neq x)$, for every term $t[x]$ containing $x$ but distinct from $x$.

5. $\square\left(\left(x_{1} \neq y_{1}\right) \vee \ldots \vee\left(x_{n} \neq y_{n}\right) \rightarrow f\left(x_{1}, \ldots, x_{n}\right) \neq f\left(y_{1}, \ldots, \downarrow\right)\right)$, for every function symbol $f$.

6. $\square(x=x)$.

7. $\square\left(\left(x_{1}=y_{1}\right) \wedge \ldots \wedge\left(x_{n}=y_{n}\right) \rightarrow f\left(x_{1}, \ldots, x_{n}\right)=f\left(y_{1}, \ldots, y_{n}\right)\right)$, for every function symbol $f$.

8. $\square\left(\left(x_{1}=y_{1}\right) \wedge \ldots \wedge\left(x_{n}=y_{n}\right) \rightarrow\left(p\left(x_{1}, \ldots, x_{n}\right) \rightarrow p\left(y_{1}, \ldots, \eta_{h}\right)\right)\right)$, for every predicate symbol $p$ (and for the predicate symbol of equality too).

Let $P$ be a normal program. The completion of infiniteness of $P, \operatorname{comp} f(P)$, is the collection of all the completed definitions of infiniteness of the predicate symbols of $\mathscr{L}$ together with the modal equational theory. Let $G$ be the normal goal $\leftarrow L_{1}, \ldots, L_{n}$ and $\theta$ a substitution of the variables of $G$. We will say that $\theta$ is a correct answer for $\operatorname{comp}_{I}(P) \cup\{G\}$ if $\exists\left(\left(\square L_{1}{ }^{*} \vee \ldots \vee \square L_{n}{ }^{*}\right) \theta\right)$ is a valid consequence of $c o m p_{I}(P)$. This notion of correct answer is the semantical counterpart of the procedural notion of a computed answer of infiniteness.

Now we define a mapping $T_{P}^{J}$ on the lattice of interpretations (based on some preinterpretation $J$ of the language) to itself. If $J$ is a classical pre-interpretation of $\mathscr{L}$ and if $I$ is a classical interpretation based on $J$ then $T_{P}{ }^{J}(I)=\{B$ : for some variable assignment $V$ with respect to $J$, there is a clause $A \leftarrow L_{1}, \ldots, L_{n}$ in $P$ such that $A_{J, V}=B$ and $L_{1}{ }^{*} \vee \ldots \vee L_{n}{ }^{*}$ is true with respect to $I$ and $\left.V\right\}$. When $J$ is the classical Herbrand preinterpretation, we will write $T_{P}$ instead of $T_{P}^{J}$. The mapping $T_{P}^{J}$ possesses some properties. 
proposition 4.1 For every pre-interpretation $J$ of $\mathscr{L}$ and for every normal program $P$, the mapping $T_{P}{ }^{J}$ is continuous.

Note that, for every program $P$, lfp $\left(T_{P}^{J}\right)=\varnothing$. In other respects, it is not always true that $g f p\left(T_{P}\right)=T_{P} \downarrow \omega$. If $P=\{A \leftarrow B(x), B(f(x)) \leftarrow B(x)\}$, then $g f p\left(T_{P}\right)=\varnothing$ and $T_{P} \downarrow \omega=$ $\{A\}$. A first important result is that the domain of interpretation of the terms of the language is not essential.

theorem 4.2 Let $P$ be a normal program and $G$ the normal goal $\leftarrow L_{1}, \ldots, L_{k}$. Let $\theta$ be a substitution of the variables in $G$. The following assertions are equivalent:

(a) $\theta$ is a correct answer for comp $(P) \cup\{G\}$, that is to say: $\exists\left(\square L_{l}{ }^{*} \theta \vee . . . v \square L_{k}{ }^{*} \theta\right)$ is true in every model of $\operatorname{comp}_{\boldsymbol{N}}(P)$.

(b) $\exists\left(\square L_{l}{ }^{*} \theta \vee . . . v \square L_{k}{ }^{*} \theta\right)$ is true in every Herbrand model of comp $p_{I}(P)$.

The proof of theorem 4.2 is not essential for our purpose. It could be done by induction on the model of $c o m p_{I}\left(P \cup\left\{\neg \exists\left({ }_{1} L_{1}{ }^{*} \theta \vee \ldots \vee \square L_{k}{ }^{*} \theta\right)\right\}\right.$. See [6] for the exact details. The result of theorem 4.2 will greatly simplified the presentation of future proofs. Especially the soundness proof of the infiniteness of SLDNF resolution we present now.

theorem 4.3 Let $P$ be a normal program and $G$ the nomal goal $\leftarrow L_{1}, \ldots, L_{k}$. Every computed answer of infiniteness of $P \cup\{G\}$ is a correct answer for comp $\{(P) \cup\{G\}$.

proof Let $\theta$ be a substitution of the variables of $G$ which is not a correct answer for comp $p_{I}(P) \cup\{G\}$. Theorem 4.2 says there is a Herbrand model of $\operatorname{comp}_{I}(P)\left\{\neg \exists\left(\square L_{l}{ }^{*} \theta \vee \ldots \vee \vee \square L_{k}{ }^{*} \theta\right)\right\}$. Let $I$ be a Herbrand interpretation with universe $M$ and accessibility relation $R$ which is a model of $\operatorname{comp}_{I}(P) \cup\left\{\neg \exists\left(\square L_{1}{ }^{*} \theta \vee \ldots \vee \square L_{k}{ }^{*} \theta\right)\right\}$. Let $w$ be a possible world of $M$ where $\operatorname{comp}_{I}(P)\left\{\neg \exists\left(\square L_{l}{ }^{*} \theta \vee \ldots \vee \square L_{k}{ }^{*} \theta\right)\right\}$ is true and such that, for every possible world $w^{\prime}$ accessible from $w$ using $R$, for every normal goal $\leftarrow l_{1}, \ldots, l_{q}$ and for every substitution $\sigma$

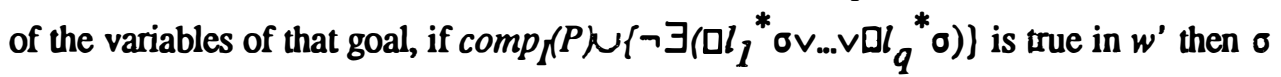
is not a computed answer of infiniteness of $P \cup\left\{\leftarrow l_{1}, \ldots, l_{q}\right\}$. Now for every $j=1, \ldots, k$ 
and for every variable assignment $V$ with respect to the Herbrand pre-interpretation, there is a possible world $w_{j, V}$ accessible from $w$ and where $c o m p_{1}(P) \cup\left\{\neg L_{j}^{*} \theta\right\} \cup\left\{\forall x_{1} \ldots \forall x_{n}\left(p_{j}\left(x_{1}, \ldots, x_{n}\right) \leftarrow E_{1} \vee \ldots \vee E_{k}\right)\right\}$ is true with respect to $V, p_{j}$ being the predicate symbol of the atom $L_{j}{ }^{*}$. Let: $L_{j}{ }^{*} \theta=p_{j}\left(t^{\prime}{ }_{1} \ldots . ., t_{n}^{\prime}\right)$. Thus, for every clause $p_{j}\left(t_{1}, \ldots, t_{n}\right) \leftarrow l_{1}, \ldots, q$ in $\left.P, \exists y_{1} . . \exists \psi_{d}\left(\left(\square l_{1} \vee \ldots \vee \vee l_{q}\right)\right) \wedge\left(t_{1}=t^{\prime}{ }_{1}\right) \wedge \ldots \wedge\left(t_{n}=t^{\prime}{ }_{n}\right)\right)$ is false in $w_{j}, V$ with respect to $V$. Let $\sigma$ be an $m g u$ of $p_{j}\left(t_{1}, \ldots, t_{n}\right)$ and $p_{j}\left(t_{1}^{\prime}, \ldots, t_{n}^{\prime}\right)$.

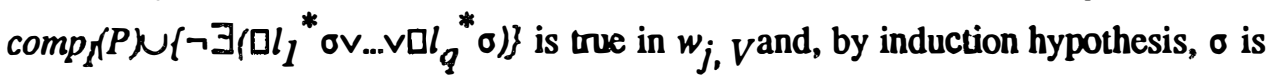
not a computed answer of infiniteness of $P \cup\left\{\leftarrow l_{1}, \ldots, l_{q}\right\}$. Consequently, $\theta$ is not a computed answer of infiniteness of $P \cup\left\{\leftarrow L_{1}, \ldots L_{k}\right\}$.

corollary 4.4 If there is an infinite SLDNF computation of $P \cup\{G\}$ then $\operatorname{comp}_{I}(P) \cup\left\{\leftarrow \exists\left(\mathrm{L}_{1}^{*} \vee \ldots \vee v \mathrm{~L}_{n}^{*}\right)\right\}$ is insatisfaisable.

corollary 4.5 The infiniteness set of $P$ is contained in the set $\left\{A \in B_{\mathscr{L}}: \square A\right.$ is a valid consequence of $\left.\operatorname{comp}_{[}(P)\right\}$.

Now we give the point of view of the fixpoint operator.

theorem 4.6 Let $P$ be a normal program and $G$ the normal goal $\leftarrow L_{1}, \ldots, L_{m}$. If $\theta$ is a computed answer of infiniteness of rank $n$ of $P \cup\{G\}$ then $\cup_{j=1 . . m}\left[L_{j}^{*} \theta\right] \cap T_{P} \downarrow n \neq \varnothing$.

proof The following induction on $n$ proves that if $\cup_{j=1 . . m}\left[L_{j}^{*} \theta\right] \subseteq B_{\mathscr{L}} \mid T_{P} \downarrow n$ then $\theta$ is a computed answer of finiteness of rank $n$ of $P \cup\{G\}$. Suppose it is true for $\alpha-1$. Let $n=\alpha$. If $\cup_{j=1 . . m}\left[L_{j}{ }^{*} \theta\right] \subseteq B \mathscr{L} \backslash T_{P} \downarrow n$ then, for every $L_{j} \theta$ in $G \theta$ and for every substitution $\sigma$ such that $L_{j} \theta \sigma$ is ground, $L_{j}{ }^{*} \theta \sigma \notin T_{P} \downarrow n$, that is to say: for every variable assignment $V$ with respect to the Herbrand pre-interpretation and for every clause $B \leftarrow l_{1}, \ldots, q$ in $P$, if $B_{V}=B_{j} \theta \sigma$ then $l_{1}^{*} v_{\text {...v }} l_{q}{ }^{*}$ is false with respect to $T_{P} \downarrow \alpha-1$ and $V$. Thus, for every clause $B \leftarrow l_{1}, \ldots q q$ in $P$ and for every substitution $\sigma$, if $\sigma=$ $m g u\left(B, B_{j} \theta\right)$ then $\cup_{k=1 . . q}\left[l_{k}^{*} \sigma\right] \subseteq B \mathscr{L} \mid T_{P} \downarrow \alpha-1$ and, by induction hypothesis, $\sigma$ is a computed answer of finiteness of rank $\alpha-1$ of $P \cup\left\{\leftarrow l_{1} \ldots . ., q\right\}$. Consequently, $\theta$ is a computed answer of finiteness of rank $n$ of $P \cup\{G\}$. 
note Theorem 4.6 does not imply that if there is an infinite SLDNF computation of $P \cup\left\{\leftarrow L_{1}, \ldots, L_{m}\right\}$ then $\cup_{j=1 . . m}\left[L_{j}^{*}\right] \cap \mathrm{T}_{P} \downarrow \omega \neq \varnothing$. As a matter of fact, if $P=$ $\{A(s(x)) \leftarrow A(x)\}$ and $G=\leftarrow A(y)$ then $\cup_{j=1 . . m^{[}}\left[L_{j}^{*}\right]=\left\{A\left(s^{n}(0)\right): n 0\right\}$ and $\mathrm{T}_{P} \downarrow \omega=\varnothing$.

corollary 4.7 The set of infiniteness of a normal program $P$ is contained in $T_{P} \downarrow \omega$.

As for the completeness proof of the infiniteness of SLDNF resolution, it has boen done using the fact that $S L D N F$ trees are finitely branching: if $P \cup\{G\}$ is allowed and if there is no infinite $S L D N F$ computation of $P \cup\{G\}$ then every $S L D N F$ tree of $P \cup\{G\}$ is finite. More precisely: some integer is greater than the depth of $S L D N F$ trees of $P \cup\{G\}$.

theorem 4.8 Let $P$ be a normal program and $G$ the normal goal $\leftarrow L_{1}, \ldots, L_{m}$. If $P \cup\{G\}$ is allowed and if $\operatorname{comp}_{I}(P) \cup\left\{\leftarrow \exists\left(\square L_{I}{ }^{*} v_{\text {... }} \vee \square L_{n}{ }^{*}\right)\right\}$ is insatisfaisable then there is an infinite SLDNF computation of $P \cup\{G\}$.

proof If there is no infinite derivation of $P \cup\{G\}$ then some integer max is greater than the length of any $S L D N F$ derivation of $P \cup\{G\}$. The proof is straightforward and can be done by induction on $\max$.

proposition 4.9 The set of infiniteness of an allowed normal program $P$ is equal ${ }_{\text {to }} \downarrow \downarrow \omega$ and is equal to the set $\left\{A \in B_{\mathscr{L}}: \square A\right.$ is a valid consequence of comp $\{(P)\}$.

proof If $A \notin T_{P} \downarrow \omega$ then, for some integer $n, A \notin T_{P} \downarrow n$. The proof is straightforward and can be done by induction on $n$.

\section{Recursive Computation Rules}

We have just characterized in provability modal logic a property of infiniteness of $S L D N F$ resolution: if $P$ is allowed then there is an infinite $S L D N F$ computation of $P \cup\{\leftarrow A\}$ if and only if $\square A$ is a valid consequence of $\operatorname{comp}_{f}(P)$ in the class of modal interpretations whose accessibility relation is transitive and reverse well-founded. This characterization does not give us a choice procedure of an atom in a goal such that if there is an infinite $S L D N F$ computation of $P \cup\{G\}$ then there is an infinite $S L D N F$ 
derivation which uses this procedure: validity in the class of transitive and reverse wellfounded interpretations is $\Pi_{2}{ }^{1}$-complete [9]. Our characterization does not say however that such a procedure cannot exist. Now the question is to see whether there could be an algorithm of selection of atoms in goals always leading into an infinite derivation when such a derivation exists. Such an algorithm is a recursive maximal computation rule for the infiniteness of SLDNF resolution. Considering definite logic programs and SLD resolution, we will show that such a rule cannot exist.

A computation rule is maximal for the refutation of $S L D$ resolution when, for every definite program $P$ and for every definite goal $G$, if there is an $S L D$ refutation of $P \cup\{G\}$ then there is an $S L D$ refutation of $P \cup\{G\}$ using this rule. An essential result of the theory of logic programming is the independence of the computation rule for the refutation of $S L D$ resolution, that is to say: every computation rule is maximal for the refutation of $S L D$ resolution [14]. A computation rule is maximal for the finite failure of $S L D$ resolution when if there is a finitely failed $S L D$ tree of $P \cup\{G\}$ then the $S L D$ tree of $P \cup\{G\}$ using this rule is finitely failed. An important result is the independence of the computation rule, as far as it is fair, for the finite failure of $S L D$ resolution [12]. Similarly, it is not difficult to prove that every fair computation rule is maximal for the finiteness of $S L D$ resolution.

Now we consider the maximality of a computation rule with respect to the infiniteness of $S L D$ resolution. A computation rule is maxinal for the infiniteness of $S L D$ resolution when, for every program $P$ and for every goal $G$, if there is an infinite $S L D$ tree of $P \cup\{G\}$ then the $S L D$ tree of $P \cup\{G\}$ using this rule is infinite. Some rules can be maximal for the infiniteness of $S L D$ resolution. A computation rule is recursive if it is an algorithm for the selection of an atom in a goal. Such rules cannot be maximal for the infiniteness of $S L D$ resolution.

theorem 5.1 There is no recursive computation rule maximal for the infiniteness of SLD resolution.

proof As a matter of fact, we prove that, for some definite program $P$, there can be no recursive computation rule maximal for the infiniteness of $S L D$ resolution in $P$. The proof is based on an idca developed by Shepherdson [17] who proved that no recursive rule can be maximal for the refutation of $S L D N F$ resolution. Let $A$ and $B$ be two recursively enumerable recursively inseparable disjoint sets [15]. Let $f$ and $g$ be two unary functions enumerating $A$ and $B$. Let $F$ and $G$ be the partial recursive functions defined as follows: $F(x)=\mu y(f(y)=x) ; G(x)=\mu y(g(y)=x) . F(x)$, respectively: $G(x)$, is, 
when it exists, the least integer $y$ such that $f(y)=x$, respectively: $g(y)=x$. Now, $F(x)$ is defined if and only if $x \in A$ and $G(x)$ is defined if and only if $x \in B$. Let $P_{F}$ be the imperative program using the variables $X_{F}$ and $Y_{F}$, and instructions like:

(a) [i] $X_{F}:=X_{F}+1$

(b) [j] IF $X_{F} O$ THEN $X_{F}:=X_{F}-1$ AND GOTO [j]

for $X_{F}$ and similar instructions for $Y_{F}$, and such that, for every input $\left(X_{F}, Y_{F}\right)=(x, 0)$, the program stops if and only if $F(x)$ is defined. Let $P_{F^{*}}$ be the logic program obtained from $P_{F}$ as follows:

(1) Replace every instruction of type (a) by the clause $p_{F, i}(X, Y) \leftarrow p_{F, i+1}(s(X), Y)$.

(2) Replace every instruction of type (b) by the clauses $p_{F}(s(X), Y) \leftarrow p_{F, j}(X, Y)$ and $p_{F}(0, Y) \leftarrow p_{F, j+I}(0, Y)$.

Let $P_{F^{* *}}$ and $P_{G^{* *}}$ be the programs obtained from $P_{F^{*}}$ and $P_{G^{*}}$ respectively by the addition of the clauses $\left.p_{F,} f^{*}(X), X\right)<-p_{F, I}(X, 0)$ and $p_{G, O}\left(G^{*}(X), X\right) \leftarrow p_{G, I}(X, 0)$. Let $P$ be the program $P_{F^{* *}} \cup P_{G^{* *}}$. Let $G_{n}$ be the goal $\leftarrow P_{F,}(X, n), p_{G, 0}(X, n)$. If we want to find an infinite $S L D$ derivation, we have the choice of the selection of an atom at the first step of the computation only. If we choose the first atom then there is an infinite $S L D$ derivation if and only if $n \notin A$. Otherwise, there is an infinite $S L D$ derivation if and only if $n \notin B$. Let $R$ be a recursive computation rule. It corresponds to a recursive set $C$ such that $R$ selects the first atom of $G_{n}$ if and only if $n \in C$. Thus, if $R$ is maximal for the infiniteness of $S L D$ derivation then $A \subseteq \mathbb{N} I C$ and $B \subseteq C$, which is impossible since $A$ and $B$ are recursively inseparable.

\section{Conclusion}

We have given a semantical characterization of finite and infinite SLDNF derivations. The completion formulas we have defined were both sound and complete for the finiteness and infineteness of SLDNF resolution: if $P$ is allowed then there is no (an) infinite $S L D N F$ computation of $P \cup\{\leftarrow A\}$ if and only if $A(\square A)$ is a valid consequence of $\operatorname{comp}_{F}(P)\left(\operatorname{comp}_{\Gamma}(P)\right)$ in classical first order predicate calculus (in transitive and reverse well-founded modal interpretations). The characterization of infinite $S L D N F$ computations that was presented in theorems 4.3 and 4.8 constitutes a first step towards 
a better understanding of the modal semantics of perpetual processes.

However, the result stated in theorem 5.1 is not very encouraging from the point of view of using PROLOG for concurrent applications. As a matter of fact, what is asked to a perpetual processes is to carry a computation which never ends. This computation has to be defined with the help of a recursive rule. As we have proved, such a nule cannot exist. Consequently, we have to circumscribe classes of definite logic programs for which recursive rules maximal for the infiniteness of $S L D$ resolution exist. The notion of a perpetual processes makes sense for these classes of programs only. Now the question is the nature of these classes of programs.

\section{Notes}

(1) 58 avenue de la république, 93110 Rosny-sous-bois, France

(2) Including the binary predicate symbol of equality.

(3) This is a direct consequence of König's lemma.

\section{References}

1. K. R. Apt, G. Bezem: Acyclic programs. In: P. Szeredi, D. H. D. Warren (eds.): Proceedings of the Seventh International Conference on Logic Programming. Massachussets Institute of Technology Press 1990, pp. 617633

2. K. R. Apt, H. A. Blair, A. Walker: Towards a theory of declarative knowledge. In: J. Minker (ed.): Foundations of Deductive Databases and Logic Programming. Los Altos: Morgan Kaufmann 1988, pp. 89-148

3. K. R. Apt, R. N. Bol, J. W. Klop: On the power of subsumption and context checks. In: A. Miola (ed.): Design and Implementation of Symbolic Computation Systems. Lecture Notes in Computer Science 429. Berlin: Springer 1990, pp. 131-140

4. P. Balbiani: A modal semantics for the negation as failure and the closed world assumption rules. In: C. Choffrut, M. Jantzen (eds.): Eighth Symposium on Theoretical Aspects of Computer Science. Lecture Notes in Computer Science 480. Berlin: Springer 1990, pp. 523-534

5. P. Balbiani: A modal semantics of negation in logic programming. Fundamenta Informatica (to appear)

6. P. Balbiani: Sur la finitude des dérivations de la programmation en logique. rapport IRIT/92-3-R

7. M. Bezem: Characterizing termination of logic programs with level 
mapping. In: E. L. Lusk, R. Overbeek (eds.): Proceedings of the North American Conference on Logic Programming. Massachussets Institute of Technology Press 1989, pp. 69-80

8. G. Boolos: The unprovability of inconsistency. Cambridge: Cambridge University Press 1979

9. G. Boolos, G. Sambin: Provability: the emergence of a mathematical modality. Studia Logica, 1-23 (1991)

10. K. L. Clark: Negation as failure. In: H. Gallaire et J. Minker (eds.): Logic and Data Bases. New York: Plenum Press 1978, pp. 293-322

11. F. Denis: Contribution à l'étude des sémantiques axiomatiques de Prolog. thèse de l'université des sciences et techniques de Lille Flandres Artois, 1990

12. J.-L. Lassez, M. J. Maher: Closures and faimess in the semantics of programminglogic. Theoretical Computer Science 29, 167-184 (1984)

13. J.-L. Lassez, M. J. Maher, D. A. Wolfram: A unified trearment of resolution strategies for logic programs. In: S.-A. Tarnlund (ed.): Second Intemational Conference on Logic Programming. Uppsala: Uppsala University Press 1984, pp. 263-276

14. J. W. Lloyd: Foundations of logic programming. Berlin: Springer 1987

15. P. Odifreddi: Classical recursion theory. Amsterdam: North-Holland 1989

16. L. Plümer: Termination proofs for logic programs. Lecture Notes in Artificial Intelligence 446. Berlin: Springer 1990

17. J. C. Shepherdson: Unsolvable problems for SLDNF resolution. Journal of Logic Programming 10, 19-22 (1991)

18. J. D. Uliman, A. van Gelder: Efficient tests for top-down termination of logical nules. Journal of the Association for Computing Machinery 35, 345373 (1988) 